\title{
Basic functionality of a prototype wearable assistive soft exoskeleton for people with gait impairments - a case study
}

\author{
Eveline S. Graf* \\ Zurich University of Applied \\ Sciences, Switzerland \\ Adam de Eyto \\ University of Limerick \\ Ireland \\ Matteo Sposito \\ Istituto Italiano di Tecnologia \\ Genova, Italy \\ Carole Pauli \\ Zurich University of Applied \\ Sciences, Switzerland \\ Leonard O’Sullivan \\ University of Limerick \\ Ireland
}

\author{
Christoph M. Bauer \\ Zurich University of Applied \\ Sciences, Switzerland \\ Eliza Bottenberg \\ Saxion University of Applied \\ Sciences, Enschede, Netherlands \\ Daniel Scherly \\ Zurich University of Applied \\ Sciences, Switzerland \\ Laura Erkens \\ Saxion University of Applied \\ Sciences, Enschede, Netherlands \\ Markus Wirz \\ Zurich University of Applied \\ Sciences, Switzerland \\ Jesús Ortiz \\ Istituto Italiano di Tecnologia \\ Genova, Italy
}

\author{
Valerie Power \\ University of Limerick \\ Ireland
}

\author{
Tommaso Poliero \\ Istituto Italiano di Tecnologia \\ Genova, Italy \\ René Henke \\ Zurich University of Applied \\ Sciences, Switzerland \\ Ger Brinks \\ Saxion University of Applied \\ Sciences, Enschede, Netherlands \\ Konrad S. Stadler \\ Zurich University of Applied \\ Sciences, Switzerland
}

\begin{abstract}
XoSoft is a soft modular wearable assistive exoskeleton for people with mild to moderate gait impairments. It is currently being developed by a European Consortium (www.xosoft.eu) and aims to provide tailored and active lower limb support during ambulation. During development, user-centered design principles were followed in parallel with the aim of providing functional support during gait. A prototype was developed and was tested for practicability, usability, comfort and assistive function (summarized as basic functionality) with a potential end user. The prototype consisted of a garment, electromagnetic clutch-controlled elastic bands supporting knee- and hip flexion and a backpack containing the sensor and actuator control of the system. The participant had experienced a stroke and presented with unilateral impairment of the lower and upper extremities. In testing, he donned and doffed the
\end{abstract}

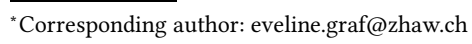

Permission to make digital or hard copies of all or part of this work for personal or classroom use is granted without fee provided that copies are not made or distributed for profit or commercial advantage and that copies bear this notice and the full citation on the first page. Copyrights for components of this work owned by others than the author(s) must be honored. Abstracting with credit is permitted. To copy otherwise, or republish, to post on servers or to redistribute to lists, requires prior specific permission and/or a fee. Request permissions from permissions@acm.org. PETRA '18, June 26-29, 2018, Corfu, Greece

(C) 2018 Copyright held by the owner/author(s). Publication rights licensed to the Association for Computing Machinery.

ACM ISBN 978-1-4503-6390-7/18/06 ...\$15.00

https://doi.org/10.1145/3197768.3197779 prototype independently as far as possible, and performed walking trials with the system in both active (powered on) and passive (powered off) modes. Afterwards, the participant rated the perceived pressure and various elements of usability. Results highlighted aspects of the system for improvement during future phases of XoSoft development, and also identified useful aspects of prototype design to be maintained. The basic functionality of XoSoft could be assumed as satisfactory given that it was the first version of a working prototype. The study highlights the benefits of this participatory evaluation design approach in assistive soft robotics development.

\section{CCS CONCEPTS}

- Social and professional topics $\rightarrow$ People with disabilities; • Applied computing $\rightarrow$ Life and medical sciences; Health care information systems; - Computing methodologies $\rightarrow$ Control methods;

\section{KEYWORDS}

exoskeleton; exosuit; soft robotics; stroke; basic functionality

\section{ACM Reference Format:}

Eveline S. Graf, Christoph M. Bauer, Valerie Power, Adam de Eyto, Eliza Bottenberg, Tommaso Poliero, Matteo Sposito, Daniel Scherly, René Henke, Carole Pauli, Laura Erkens, Ger Brinks, Leonard O'Sullivan, Markus Wirz, Konrad S. Stadler, and Jesús Ortiz. 2018. Basic functionality of a prototype wearable assistive soft exoskeleton for people with gait impairments - a case study. In PETRA '18: The 11th PErvasive Technologies Related to Assistive 
Environments Conference, fune 26-29, 2018, Corfu, Greece. ACM, New York, NY, USA, 6 pages. https://doi.org/10.1145/3197768.3197779

\section{INTRODUCTION}

Gait impairment and the need for mobility assistance is commonly observed in people with different health conditions, such as residual paresis after stroke, incomplete spinal cord injury, and general age-related frailty. With the expected increase of the world population aged 60 years and over [18] and the increasing rate of survival after experiencing a stroke [7] it can be expected that the number of people in need of low to moderate mobility assistance will continue to increase. Wearable assistive devices such as soft exoskeletons are possible solutions for these conditions.

With this in mind, the XoSoft project (www.xosoft.eu) is developing one such solution to address this challenge. XoSoft is not intended to substitute complete loss of function as is already the target of other exoskeletons (e.g. ReWalk, Ekso Bionic), but rather assist users with partial mobility limitations actively. For the development of XoSoft, a user-centered design approach was chosen to ensure that the expectations of potential end users were met, as far as is technically feasible [15]. Previous work has identified some specific design requirements for a soft exoskeleton such as XoSoft. Independent use, for example easy donning and doffing also for people with upper limb impairments, comfortable wear, and compatibility with usual footwear and clothing are some of the aspects defined by end users and health care professionals as being important to address during development [15]. The function of XoSoft also needs to be specific to the functional limitation of the person wearing the exosuit and provide the necessary support. Challenges are associated with such requirements - exosuits would most likely have to be customized to the individual user, and adjustable modular function should be possible so that appropriate support can be provided to the joints in need of support.

Since XoSoft is a wearable device, the mechanical loading of human tissue needs to be considered. When wearing an assistive device, contact and friction forces act between the device and the skin, and the magnitude of these forces is crucial to consider if the design is to be comfortable to wear. In particular, if the device is used by people with neurological conditions, who often experience sensory impairments, soft tissue injury, such as pressure sores, may easily develop as a consequence of inappropriate design [14]. Besides the load magnitude, other load characteristics, such as direction, distribution, duration (known as the '3Ds'), and loading rate should be considered in the discussion of soft tissue responses to external loads [9]. An assistive device such as XoSoft is intended to be worn for long durations meaning that only low pressures can be tolerated [17].

XoSoft is currently being developed and a first prototype had been completed. Based on this prototype, aspects of practicability and usability, comfort and assistive function, summarized as basic functionality, were tested with a potential end user. The aim of this case study was to assess basic functionality of the prototype and inform future development of XoSoft.

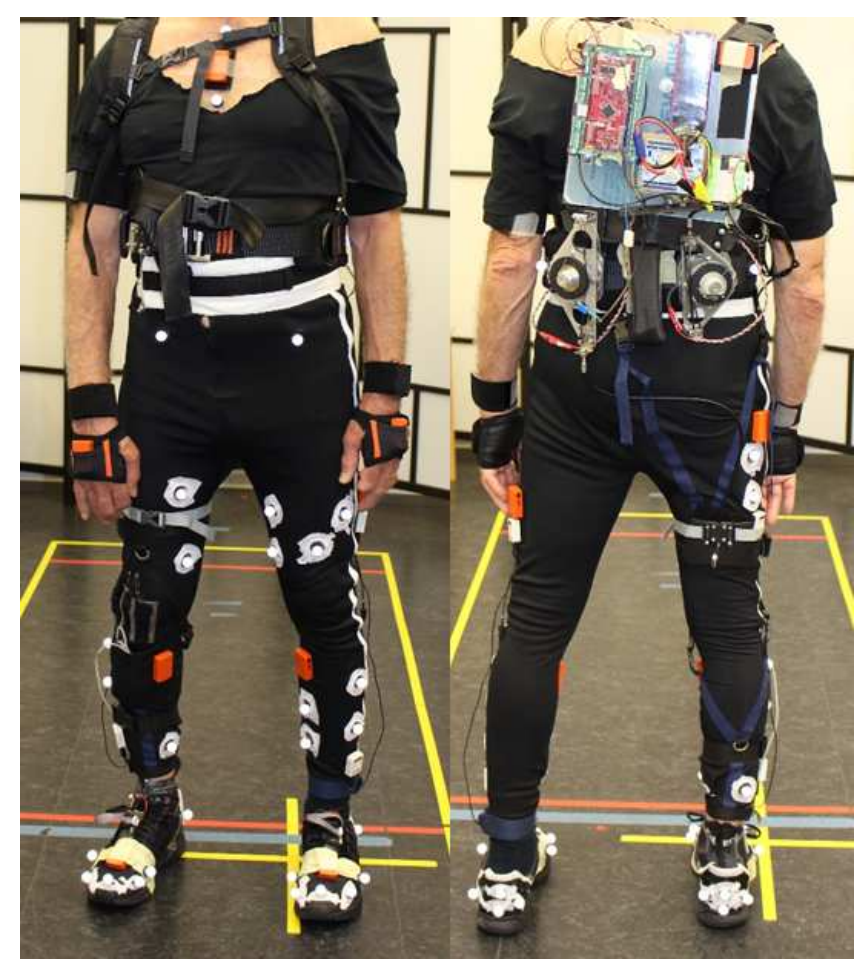

Figure 1: Participant wearing XoSoft (view from front and back)

\section{METHODS}

\subsection{Description of XoSoft Prototype}

The XoSoft prototype consisted of a garment that was customfitted for the participant, two clutch-controlled elastic bands (ventral to the hip and dorsal to the knee), as well as a separate belt where the electromagnetic clutches were attached (Figure 1). Additionally, the participant wore a backpack containing a central processing unit (Launchpad XL Development Kit, Texas Instruments, Dallas, USA) that controlled the system. This board was coupled with a custom shield board for advanced communication and power management.

For the garment, a knitted stretchable textile fabric with quick drying properties was chosen. A Cummerbund style hip belt combined with two ribbon webbings was used to anchor the garment above the iliac crests. Additional ribbon webbing was added to provide some tension and to support the elements of the garment. The ribbon webbing was combined with squeeze buckles as closure mechanisms. To facilitate donning and doffing, full-length zippers were integrated on the lateral aspects of both legs.

The elastic bands (Loop Band, Fit Point, Brescia, Italy) at the hipand knee-joints were designed to support joint flexion in the initial swing phase. The elastic bands were connected to electromagnetic clutches (111-06-13G, Miki Pulley Co. Ltd, Kanagawa, Japan) using Bowden cables for the system to store mechanical energy during the stance phase, and to release the energy during swing phase. The engagement or disengagement of the clutches determined how 
much energy was being stored. Indeed, if the clutches were disengaged, the Bowden cable was free to travel and the elastic band, connected in series, did not experience any elongation. Otherwise, as a consequence of the clutch engagement, the bowden cable was not free to travel anymore and, hence, the user joint rotations resulted in elastic elongation [13].

The participant wore a commercially available running shoe (Nike Air Zoom Pegasus 33). The insoles of the shoe were replaced with custom made pressure sensitive insoles, comprising of four standard force sensing resistors (FSR) (1-Inch ShuntMode, Sensitronics, Bow, USA). The FSRs were arranged underneath the heel, the $1^{\text {st }}$ and $5^{\text {th }}$ metatarsophalangeal joints, and the distal phalanx of the big toe. The FSR signals were used to detect four different phases of gait: initial contact, flat foot, final contact, and swing. Based on these phases, specific control signals for the electromagnetic clutches were triggered. When the foot was flat, the hip- and knee-clutch engaged leading to no further elongation of the Bowden cable. The elastic bands were stretched when the joints moved further into extension. $140 \mathrm{~ms}$ after swing phase was detected, both clutches disengaged and released the Bowden cables.

\subsection{Participant}

The participant was a 68 year old male (mass: $72 \mathrm{~kg}$; height: $170 \mathrm{~cm}$ ) who experienced a cerebrovascular stroke seven years ago. He presented with unilateral impairment of the upper and lower extremity with the right side affected. In everyday life, the participant uses a single walking stick and was classified with a Functional Ambulation Category of 4 [10]. The local ethics committee approved the study and the participant signed informed written consent prior to taking part.

\subsection{Tasks/Conditions}

After donning XoSoft independently (as far as possible), the participant performed ten walking trials for each condition. First, he walked with the condition with no elastic bands (None), followed by the condition with the elastic bands (XoSoft). The participant was instructed to walk at a self-selected speed during the None condition. The same speed was used for the XoSoft condition. Speed was monitored using two photocell timing lights (Witty, Microgate, Bolzano, Italy). After the walking trials, the participant doffed XoSoft independently.

\subsection{Measurement Systems and Data Analysis}

Three or four retro-reflective markers were attached to each of the relevant body segments (forefoot, rearfoot, shank, thigh, pelvis, trunk). These markers were recorded with a 3D highspeed 12 camera system (Vicon Vantage V5, Vicon Motion Systems Ltd, Oxford, UK) at a sampling rate of $240 \mathrm{~Hz}$. Before analysis, data were filtered using a low-pass Butterworth filter $\left(4^{\text {th }}\right.$-order) with a cut-off frequency of $7 \mathrm{~Hz}$. Before the dynamic trials were performed, the participant executed knee flexions and hip rotations for a modified functional calibration to determine the knee joint center and axis of rotation and hip joint center [8]. In addition, a static trial was performed with markers at additional anatomical landmarks to define the joints. A combination of the functional calibration and anatomical landmark coordinates was used to define coordinate

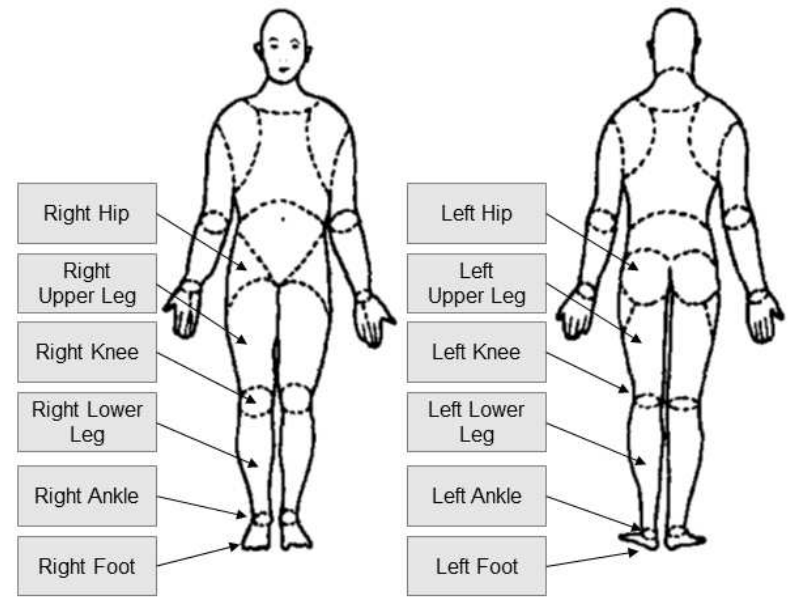

Figure 2: Body regions for which the rate of perceived pressure was collected.

systems of the segments. Joint angles were calculated based on the standards defined by the International Society of Biomechanics [5]. The chosen marker model allows for placement of the markers on the segments independently from anatomical landmarks. Also, markers do not have to be placed on the same location for the left and right side. This gives flexibility to accommodate the XoSoft prototype.

The mean and standard deviation of the ten trials were calculated, unless a trial showed missing markers during the gait cycle of interest. In this case, the trial was excluded from further analysis. All kinematic data are plotted from 0 to $100 \%$ of the gait cycle $(0 \%=$ touch-down of heel, $100 \%=$ consecutive touch-down of the same heel).

After each condition, the participant was requested in a standardized way to rate perceived pressure and system usability. To measure the perceived pressure, the lower extremities, including the pelvis, were divided into 12 distinct regions (Figure 2) [3]. The participant was requested to rate the pressure he experienced at each region on a scale from zero to ten (zero being no pressure, ten being almost maximal pressure). The system usability was rated using the System Usability Scale (SUS) (Table 1) according to [1].

\section{RESULTS AND DISCUSSION}

The purpose of this work was to analyze the basic functionality of the XoSoft prototype. Basic functionality was defined as practicability and usability, comfort and the functional assistance the system provided in terms of walking kinematics.

\subsection{Practicability: Donning/Doffing}

Some aspects of donning and doffing of the garment posed difficulties for the participant. Multiple squeeze buckles needed to be closed during donning. The buckles placed at the posterior aspect of the body caused particular difficulties when attempting to close as they were difficult to reach, especially since the participant 
Table 1: System Usability Scale

\begin{tabular}{|c|c|c|c|c|c|}
\hline Statement & 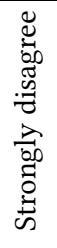 & 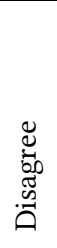 & 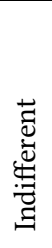 & 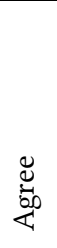 & 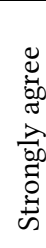 \\
\hline $\begin{array}{l}\text { 1. I think that I would like to use this } \\
\text { system frequently. }\end{array}$ & 1 & 2 & 3 & 4 & 5 \\
\hline $\begin{array}{l}\text { 2. I found the system unnecessarily } \\
\text { complex. }\end{array}$ & 1 & 2 & 3 & 4 & 5 \\
\hline $\begin{array}{l}\text { 3. I thought the system was easy to } \\
\text { use. }\end{array}$ & 1 & 2 & 3 & 4 & 5 \\
\hline $\begin{array}{l}\text { 4. I think that I would need the sup- } \\
\text { port of a technical person to be able } \\
\text { to use this system. }\end{array}$ & 1 & 2 & 3 & 4 & 5 \\
\hline $\begin{array}{l}\text { 5. I found the various functions in } \\
\text { this system were well integrated. }\end{array}$ & 1 & 2 & 3 & 4 & 5 \\
\hline $\begin{array}{l}\text { 6. I thought there was too much in- } \\
\text { consistency in this system. }\end{array}$ & 1 & 2 & 3 & 4 & 5 \\
\hline $\begin{array}{l}\text { 7. I would imagine that most people } \\
\text { would learn to use this system very } \\
\text { quickly. }\end{array}$ & 1 & 2 & 3 & 4 & 5 \\
\hline $\begin{array}{l}\text { 8. I found the system very cumber- } \\
\text { some to use. }\end{array}$ & 1 & 2 & 3 & 4 & 5 \\
\hline $\begin{array}{l}\text { 9. I felt very confident using the } \\
\text { system. }\end{array}$ & 1 & 2 & 3 & 4 & 5 \\
\hline $\begin{array}{l}\text { 10. I needed to learn a lot of things } \\
\text { before I could get going with this sys- } \\
\text { tem. }\end{array}$ & 1 & 2 & 3 & 4 & 5 \\
\hline
\end{tabular}

has unilateral upper extremity impairment. The impaired upper extremity also prevented the participant from independently closing the Cummerbund-style waist band. The full-length zippers on the lateral aspects of both legs were very useful for donning as well as doffing. Easy donning and doffing, preferable independently, have been rated as a very important feature for an assistive device [15] Consequently, the zipper feature should be maintained for future versions of XoSoft. However, the placement of buckles needs to be reconsidered and the number of buckles should be reduced to a minimum. Current developments are focusing on facilitating donning of XoSoft.

\subsection{System Usability Scale (SUS)}

The total score of the SUS was 65. Previous research has shown that a SUS total score of 65 is between "ok" and "good" for adjective rating [1]. Consequently, the score obtained for XoSoft can be deemed acceptable given the fact that this was the first iteration of the prototype. Future versions of XoSoft will also be rated with the SUS. It has been shown that the SUS is able to measure
Table 2: Rating of perceived pressure for 12 body regions

\begin{tabular}{ccccccc}
\hline Side & Foot & Ankle & Lower leg & Knee & Upper leg & Hip \\
\hline Right & 0 & 0 & 3 & 2 & 2 & 2 \\
Left & 0 & 0 & 0 & 0 & 0 & 0 \\
\hline
\end{tabular}

progress in usability of a product [1]. The obtained value can then serve as a reference point to quantify progress and future version of XoSoft can only be deemed acceptable if they reach higher total SUS scores.

\subsection{Comfort: Perceived Pressure, Pressure Points}

Table 2 shows the perceived pressure ratings obtained after the walking trials with XoSoft. Pressure was felt on the right side at the lower leg, knee, upper leg and hip. The maximum rating of 3 represents moderate pressure and was reported for the lower leg. This was most likely due to the tight anchors placed proximal to the ankle and at mid-shank height. While this rating indicates the presence of pressure, the value is not sufficiently high to cause concern. Besides the subjective rating by the participant, visual inspection of the skin after data collection showed pressure marks which were small for the left pelvis area. On the right side, some increased redness could be detected with the skin remaining intact corresponding to a stage I of the International NPUAP/EPUAP Pressure Ulcer Classification System [11]. The redness resolved after a short period. It was most likely caused by the Cummerbund style belt of the garment, which was creased during tightening of the belt containing the clutches. The combination of the Cummerbund style hip belt and the belt containing the clutches should be reconsidered, and a single integrated solution explored for future versions of XoSoft.

Pressure ulcers often start to develop in the skin and may subsequently progress to deeper tissue layers [12]. Deep ulcers arise in deep muscle layers covering bony prominences and are mainly caused by sustained compression of the tissue. These very harmful ulcers are frequently overlooked by risk-assessment techniques, most of which focus on the skin and ignore the underlying tissue [2]. However, where the tissue is subjected to abnormally high dynamic compressive stresses and shear stresses during ambulation, the skin is the first to show signs of breakdown $[6,16]$. Therefore, inspection of the skin for an early detection of stresses needs to be maintained for future prototypes of XoSoft.

\subsection{Kinematics}

At the knee joint, XoSoft increased the maximal knee flexion during swing phase by eight degrees (Figure 3). This could signify a better foot clearance during walking and, therefore, a reduced risk of tripping. A very specific characteristic of the participant's gait could be observed between 75 and $90 \%$ of the gait cycle. During this phase, the hip flexion movement on the right side (affected) was reversed into a hip extension movement before the hip started the flexion movement again at around $87 \%$ (Figure 4). The movement 


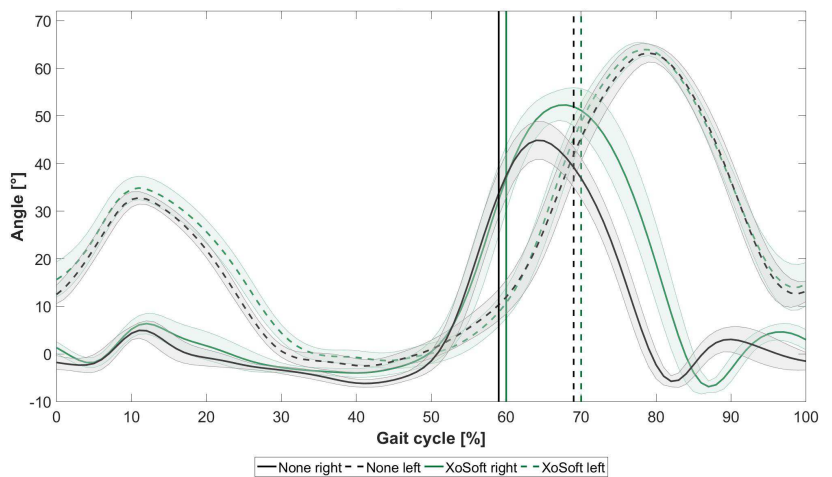

Figure 3: Knee flexion angle in degrees for None (black) and XoSoft (green) condition during walking (solid: affected/right side; dashed: unaffected/left side).

could be described as a "whipping" move that increased the forward swing of the shank and foot. The consequence of this whipping move can be seen at the knee joint. Between 80 and $90 \%$ of gait cycle, the whipping move is assumed to cause the increased knee extension on the right side. This increased knee extension is reduced by a short phase of knee flexion before the lower leg was brought into position for foot-ground contact. It can be concluded that the participant adopted this compensatory strategy to make sure that his affected knee is in sufficient extension before the weight bearing stance phase. This is most likely due to muscle weakness in the knee extensor and foot plantarflexor muscles. This movement strategy could be seen with and without XoSoft. However, when wearing XoSoft, this whipping move was more accentuated compared to the None condition. This is likely because the elastic band at the knee added resistance, making it harder to extend the knee. Additionally, maximum hip extension was reduced by XoSoft (Figure 4). Due to this reduced hip range of motion, the available time and path over which to extend the knee during hip flexion was reduced, causing the more prominent whipping move.

It can be concluded that the configuration of the elastic bands was not ideal for this specific participant. The elastic band placed at the knee aimed to restrict the whipping move. However, the participant relied on his compensatory strategy during late swing and the intended effect was not achieved. A different strategy would be necessary to support this participant with knee extension control during preparation for foot-ground contact.

The observed adaptations of the hip and knee trajectories occurred immediately and spontaneously. The present study did not investigate gait following prolonged use of XoSoft, or use following an extended period of gait training. End users may have trained and optimized their compensatory movement patterns over many years after experiencing an incident. An extended training period may be necessary for new user of XoSoft, since relearning more physiological movement patterns may require an adaptation phase that needs to be accompanied by intensive gait training [4].

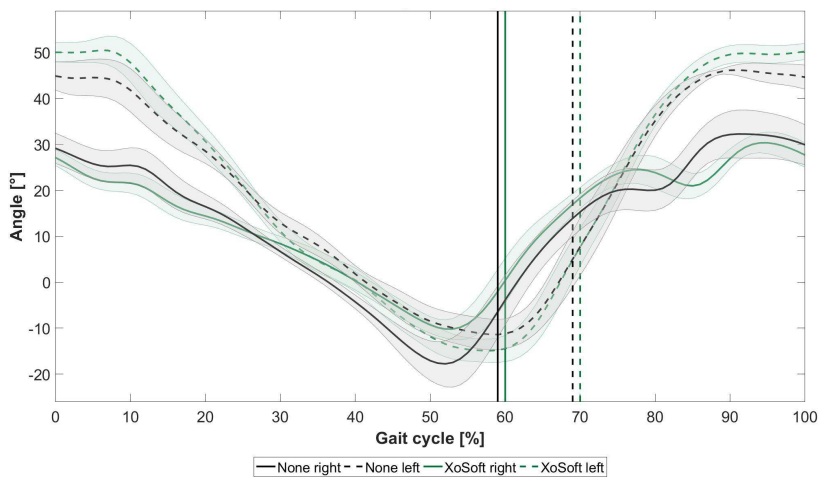

Figure 4: Hip flexion angle in degrees for None (black) and XoSoft (green) condition during walking (solid: affected/right side; dashed: unaffected/left side).

\section{CONCLUSIONS}

In summary, the basic functionality testing of XoSoft provided insights into positive aspects of the current technology design, and aspects to improve for future development to ensure it is comfortable and easy to use. Donning and doffing were facilitated by having a full-length zipper on the lateral aspect of the leg. Buckles for anchoring of actuators caused difficulties during donning. More work is needed to develop a system that is easy to don and doff for people with upper extremity impairments. The usability of the entire system was sufficient given the early stage of development of the concept, but it needs to be continuously refined through user testing and feedback so that the next iterations improve sufficiently. Further, this prototype has only been tested on a single subject. Future versions of XoSoft will be tested on a larger group of potential end users as well as in settings that represent more real-life environments. The chosen fabrics and technical solutions resulted in good comfort and can be used for future versions of XoSoft. The effectiveness of the concept in providing assistance during movement needs to be improved through more in-depth and more precisely regulated actuation solutions. Overall, the basic functionality of the first prototype of XoSoft could be assumed as satisfactory.

\section{ACKNOWLEDGMENTS}

This work has received funding from the European Union's Horizon 2020 research and innovation programme under grant agreement No. 688175 (XoSoft).

The authors would like to acknowledge the contribution of the whole XoSoft Consortium to the project and the development of the prototype.

\section{REFERENCES}

[1] A. Bangor, P.T. Kortum, and J.T. Miller. 2008. An Empirical Evaluation of the System Usability Scale. International fournal of Human-Computer Interaction 24, 6 (2008), 574-594.

[2] C. V. Bouten, C. W. Oomens, F. P. Baaijens, and D. L. Bader. 2003. The etiology of pressure ulcers: Skin deep or muscle bound? Archives of Physical Medicine and Rehabilitation 84, 4 (apr 2003), 616-619. https://doi.org/10.1053/apmr.2003.50038 
[3] J. A. Cameron. 1996. Assessing work-related body-part discomfort: Current strategies and a behaviorally oriented assessment tool. International fournal of Industrial Ergonomics 18, 5 (1996), 389-398.

[4] B. French, L. H. Thomas, J. Coupe, N. E. McMahon, L. Connell, J. Harrison, C. J. Sutton, S. Tishkovskaya, and C. L. Watkins. 2016. Repetitive task training for improving functional ability after stroke - Cochrane Database Syst Rev. Cochrane Database of Systematic Rev 11 (2016) https://doi.org/10.1002/14651858.CD006073.pub3

[5] E. S. Grood and W. J. Suntay. 1983. A Joint Coordinate System for the Clinical Description of Three-Dimensional Motions: Application to the Knee. Transactions of the ASME 105 (1983), 136-144.

[6] F.T. Hoaglund, H.E. Jergesen, L. Wilson, W. Lamoreux, and R. Roberts. 1983. Evaluation of problems and needs of veteran lower-limb amputees in the San Francisco Bay Area during the period 1977âĂŞ1980. Fournal of rehabilitation research and development 20 (1983), 57-71.

[7] K. Lakshminarayan, A. K. Berger, C. C. Fuller, D. R. Jacobs Jr, D. C Anderson, L. M. Steffen, A. Sillah, and R. V. Luepker. 2014. Trends in Ten-Year Survival of Stroke Patients Hospitalized between 1980 and 2000: The Minnesota Stroke Survey. Stroke2 45, 9 (2014), 2575-2581. https://doi.org/10.1161/STROKEAHA.114.005512.

[8] R. List, T. Gülay, M. Stoop, and S. Lorenzetti. 2013. Kinematics of the trunk and the lower extremities during restricted and unrestricted squats. Journal of strength and conditioning research 27, 6 (jun 2013), 1529-38. https://doi.org/10.1519/JSC.0b013e3182736034

[9] A. F. Mak, M. Zhang, and D. A. Boone. 2001. State-of-the-art research in lower-limb prosthetic biomechanics-socket interface: a review. Journal of rehabilitation research and development 38, 2 (2001), 161-74. http://www.ncbi.nlm.nih.gov/pubmed/11392649

[10] J. Mehrholz, K. Wagner, K. Rutte, D. Meißner, and M. Pohl. 2007. Predictive Validity and Responsiveness of the Functional Ambulation Category in Hemiparetic Patients After Stroke. Archives of Physical Medicine and Rehabilitation 88, 10 (oct 2007), 1314-1319. https://doi.org/10.1016/J.APMR.2007.06.764

[11] National Pressure Ulcer Advisory Panel, European Pressure Ulcer Advisory Panel and Pan Pacific Pressure Injury Alliance 2014. Prevention and Treatment of Pressure Ulcers: Quick Reference Guide. National Pressure Ulcer Advisory Panel, European Pressure Ulcer Advisory Panel and Pan Pacific Pressure Injury Alliance, Perth, Australia.

[12] C. W. J. Oomens, S. Loerakker, and D. L. Bader. 2010. The importance of internal strain as opposed to interface pressure in the prevention of pressure related deep tissue injury. Journal of tissue viability 19, 2 (may 2010), 35-42. https://doi.org/10.1016/j.jtv.2009.11.002

[13] J. Ortiz, T. Poliero, G. Cairoli, E. Graf, and D. G. Caldwell. 2018. Energy Efficiency Analysis and Design Optimization of an Actuation System in a Soft Modular Lower Limb Exoskeleton. IEEE Robotics and Automation Letters 3, 1 (jan 2018), 484-491. https://doi.org/10.1109/LRA.2017.2768119

[14] J.L. Pons. 2008. Wearable Robots: Biomechatronic Exoskeletons. Wiley-Blackwell, Hoboken.

[15] V. Power, L. O’Sullivan, A. de Eyto, S. Schülein, C. Nikamp, C. Bauer, J. Mueller, and J. Ortiz. 2016. Exploring User Requirements for a Lower Body Soft Exoskeleton to Assist Mobility. In Proceedings of the 9th ACM International Conference on PErvasive Technologies Related to Assistive Environments - PETRA '16. ACM New York, NY, Corfu Island, Greece, 1-6. https://doi.org/10.1145/2910674.2935827

[16] J. E. Sanders, B. S. Goldstein, and D. F. Leotta. 1995. Skin response to mechanical stress: adaptation rather than breakdown-a review of the literature. Journal of rehabilitation research and development 32, 3 (oct 1995), 214-26. http://www.ncbi.nlm.nih.gov/pubmed/8592293

[17] I. D. Swain and D. L. Bader. 2002. The measurement of interface pressure and its role in soft tissue breakdown. Fournal of tissue viability 12, 4 (oct 2002), 132-4, 136-7, 140-6. http://www.ncbi.nlm.nih.gov/pubmed/12476502

[18] World Health Organization 2014. Facts about ageing. World Health Organization http://www.who.int/ageing/about/facts/en/ Last accessed: 2018-01-19. 